\title{
Acanthamoeba and bacteria produce antimicrobials to target their counterpart
}

\author{
Junaid lqbal, Ruqaiyyah Siddiqui and Naveed Ahmed Khan*
}

\begin{abstract}
Background: In the microbial ecosystem, microbes compete for space and nutrients. Consequently, some have developed the ability to kill or inhibit the growth of other competing microbes by producing antimicrobial substances. As the 'producer' species are generally immune to these substances, their compounds act on the competing microbial species and give the producer more space and access to nutrients for growth. Many currently used antibiotics were developed by exploiting this potential of certain microbes.

Findings: Here, the free-living amoeba, Acanthamoeba castellanii, was investigated for its antibacterial activity against representative Gram positive and Gram negative bacteria, while bacterial isolates were tested for their anti-amoebic properties. Conditioned medium from A. castellanii showed remarkable bactericidal properties against methicillin-resistant Staphylococcus aureus (MRSA) exhibiting almost 100\% kill rate, but had limited effect against Acinetobacter sp., Pseudomonas aeruginosa and vancomycin-resistant Enterococcus faecalis (VRE). Similarly, the conditioned medium of E. coli $\mathrm{K} 1$ and Enterobacter sp., exhibited potent anti-Acanthamoebic effects in a concentrationdependent manner. Conditioned media of Acanthamoeba, E. coli K1 and Enterobacter sp. showed no cytotoxicity in vitro when tested against human brain microvascular endothelial cells. Active molecule/s in aforementioned amoebic and two bacterial conditioned media were $5-10 \mathrm{kDa}$, and $<5 \mathrm{kDa}$ respectively.

Conclusions: A. castellanii conditioned medium showed potent bactericidal properties against MRSA. The active molecule(s) are heat- and pronase-resistant, and in the 5 to $10 \mathrm{kDa}$ molecular mass range. Contrary to this, E. coli K1 and Enterobacter sp., conditioned medium showed anti-amoebic effects that are $<5 \mathrm{kDa}$ in molecular mass, suggestive of active metabolites.
\end{abstract}

\section{Findings}

Infectious diseases account for $68 \%$ of global child mortality, with the vast majority occurring in developing countries [1-3]. Novel antimicrobial agents are urgently needed to counter multi-drug resistant pathogens [4]. Most clinically used antibiotics are either derived from natural products especially from microbes or semisynthetic derivatives of these molecules [5-7]. In parallel, there is a need to expand the range of organisms that can be tapped in our search to discover natural product antibiotics. Given the importance of small molecules to microbial ecology, e.g., penicillin, which is produced by Penicillium as a defence mechanism [8,9], it is likely that other microbes can also contribute to the discovery of new antibiotics. Acanthamoeba is one of the most ubiquitous

\footnotetext{
* Correspondence: Naveed5438@gmail.com

Department of Biological and Biomedical Sciences, Aga Khan University, Karachi, Pakistan
}

protists that has been isolated from diverse environments [10]. In its natural habitat, i.e., soil and water, Acanthamoeba feeds on different bacteria and plays an important role in the regulation of bacterial populations [11]. However, certain bacteria especially pathogenic bacteria have developed strategies to escape the killing mechanisms of Acanthamoeba and instead use the amoeba as a Trojan horse or reservoir for their own benefit $[12,13]$. Some bacteria protect themselves from preying amoeba by secreting soluble anti-Acanthamoeba substances in their medium [14-16] or directly injecting such substances into Acanthamoeba through the type III secretory system [15]. These findings suggest that Acanthamoeba and Bacteria encounter each other in the environment routinely. But, how Acanthamoeba survive the onslaught of overwhelming bacterial population remains incompletely understood. Thus, it is reasonable to hypothesize that, in addition to bacteria, Acanthamoeba also possesses antibiotics to 
counter bacterial attack. As Acanthamoeba and bacteria thwart each other in their natural habitat, the overall aim of this study was to determine the anti-bacterial activities of Acanthamoeba and also the anti-Acanthamoebic properties of selected bacterial strains.

A keratitis isolate of Acanthamoeba castellanii was obtained from the American Type Culture Collection (50492) and grown axenically in PYG medium [proteose peptone $0.75 \%(\mathrm{w} / \mathrm{v})$, yeast extracts $0.75 \%(\mathrm{w} / \mathrm{v})$ and glucose $1.5 \%(\mathrm{w} / \mathrm{v})]$ in tissue culture flasks aerobically at $30^{\circ} \mathrm{C}$ without shaking [17-19]. A. castellanii conditioned media (ACM) were prepared by incubating confluent cultures $\left(\sim 2 \times 10^{6}\right.$ trophozoites $\left./ \mathrm{mL}\right)$ for $48 \mathrm{~h}$ in PYG. Given confluent cultures, amoebae number increased slightly to $\sim 3 \times 10^{6}$ trophozoites $/ \mathrm{mL}$. Next, cell-free medium was collected by centrifugation $(1000 \times \mathrm{g}$ for $5 \mathrm{~min})$ and filtered using $0.22 \mu \mathrm{M}$ pore size filters. In some experiments, the ACM was treated at $95^{\circ} \mathrm{C}$ for $10 \mathrm{~min}$ to inactivate $A$. castellanii extracellular proteases.

Bacterial isolates used in the present study were Acinetobacter sp., Aeromonas hydrophila, Enterobacter aerogenes, Enterobacter sp., Escherichia coli K1, Klebsiella pneumoniae, Methicillin-resistant Staphylococcus aureus (MRSA), Pseudomonas aeruginosa, Shigella flexneri, and Vancomycin-resistant Enterococcus faecalis (VRE). Escherichia coli K1 strain RS218 (O18:K1:H7) and MRSA have been described previously $[20,21]$. They were isolated from the cerebrospinal fluid of a meningitis patient [20] and blood culture of septicemia patient [21], respectively. Enterobacter sp. was isolated as a contaminant in Acanthamoeba culture, and all other bacteria are clinical isolates obtained from the Aga Khan University Hospital, Karachi (available upon request). Bacterial conditioned media (BCM) were prepared by culturing single colonies (grown on nutrient agar plates) in $100 \mathrm{~mL}$ PYG medium and incubating for $24 \mathrm{~h}$ at $37^{\circ} \mathrm{C}$ aerobically, with shaking. These conditions resulted in growth of all bacterial cultures to stationary phase of their growth. The bacterial cultures were centrifuged at $4000 \times \mathrm{g}$ at $4{ }^{\circ} \mathrm{C}$ for $20 \mathrm{~min}$. The supernatants were collected and filter sterilized using $0.22 \mu \mathrm{M}$ pore size membrane filters. In some experiments, BCM were treated at $95^{\circ} \mathrm{C}$ for $10 \mathrm{~min}$ to inactivate extracellular enzymes.

\section{Bacterial conditioned media inhibited $A$. castellanii growth}

To determine amoebistatic activity of BCM, A. castellanii trophozoites $\left(10^{5}\right.$ amoebae $/ 0.5 \mathrm{~mL} /$ well $)$ were incubated in PYG with different amounts of BCM in 24 well plates at $30^{\circ} \mathrm{C}$ for $48 \mathrm{~h}$. After this incubation, amoebae were counted using a haemocytometer. In controls, A. castellanii trophozoites were incubated in PBS (non-nutrient) and exhibited no growth (Figure 1A), while $A$. castellanii incubated in PYG (growth medium) exhibited a nine-fold increase in amoebae numbers from the original inoculum
(Figure 1A). Among the different bacterial isolates tested, Enterobacter sp., and E. coli K1 BCM inhibited Acanthamoeba growth (ca. $98 \%$ and $97 \%$ amoebistatic effects respectively). To determine whether amoebistatic effects of $\mathrm{BCM}$ were due to nutrient-depletion, A. castellanii trophozoites ( $10^{5}$ amoebae in $250 \mu \mathrm{L}$ PYG) were incubated with $250 \mu \mathrm{L}$ of PBS in a 1:1 ratio. The results revealed amoebae growth profiles, in 50\% PBS, similar to amoebae incubated in neat PYG (Figure 1A). Among other bacteria, BCM of Acinetobacter sp., A. hydrophila, K. pneumoniae and MRSA also inhibited A. castellanii growth (ca. 40\%, $35 \%, 33 \%$ and $38 \%$ amoebistatic effects respectively). The conditioned media showed amoebistatic properties, indicating the water soluble nature of the bioactive molecule(s). In order to address the possibility of conversion of Acanthamoeba trophozoites into cysts after BCM treatment, BCM-treated Acanthamoeba trophozoites were incubated with $0.5 \%$ SDS for $10 \mathrm{~min}$, which is known to selectively lyse trophozoites [22]. Post-SDS treatment, none of the BCM-treated Acanthamoeba exhibited cyst presence. To determine the potency of the compound(s) in the conditioned media of Enterobacter sp., and E. coli $\mathrm{K} 1$, serial dilutions of the $\mathrm{BCM}$ were made and tested against $A$. castellanii. The results demonstrated that $\mathrm{BCM}$ of Enterobacter sp. exhibited amoebistatic effects in a concentration-dependent manner (Figure 1B). At 50\% dilution, Enterobacter sp. BCM exhibited potent amoebistatic effects (ca. 97\%) (Figure 1B), and similarly E. coli K1 BCM exhibited more than 95\% amoebistatic effects (Figure 1B). However, Enterobacter sp. BCM was found to be more potent in inhibiting $A$. castellanii even at lower dilutions, compared with E. coli K1 BCM (Figure 1B).

\section{Enterobacter sp. and $E$. coli $\mathrm{K} 1$ conditioned media produced $A$. castellanii death}

For amoebicidal activity of BCM, A. castellanii trophozoites $\left(10^{6}\right.$ amoebae $/ 0.5 \mathrm{~mL} /$ well $)$ were incubated in PYG with different amounts of BCM in 24 well plates at $30^{\circ} \mathrm{C}$ for $48 \mathrm{~h}$. Following this incubation, amoebae viability was determined by adding 0.1\% Trypan blue and number of live (non-stained) and dead (stained) A. castellanii were enumerated using a hemocytometer. At 50\% concentration, E. coli $\mathrm{K} 1$ and Enterobacter sp. BCM did not affect $A$. castellanii viability $(0 \%$ and $0.7 \%$ respectively), while at $100 \%$ concentration, BCM of both E. coli $\mathrm{K} 1$ and Enterobacter sp. exhibited more than $90 \%$ amoebicidal effects (Figure 1C).

\section{Anti-Acanthamoebic compound(s) in Enterobacter sp. and $E$. coli $\mathrm{K} 1$ conditioned media are pronase-resistant and $<5 \mathrm{kDa}$ in molecular mass}

To determine the molecular mass of the active molecule(s), conditioned media of Enterobacter sp. and E. coli $\mathrm{K} 1$ were filtered through $10 \mathrm{kDa}$ and $5 \mathrm{kDa}$ molecular weight 


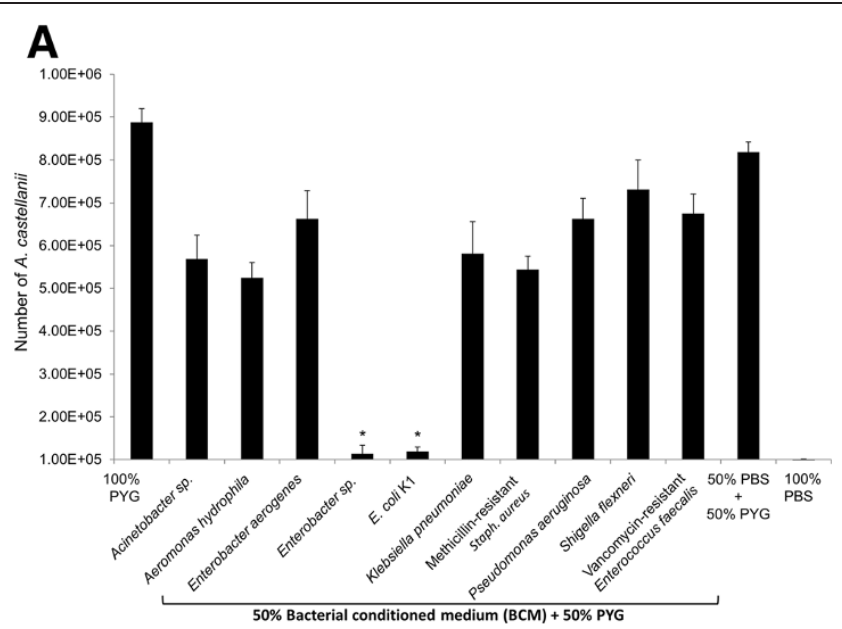

B

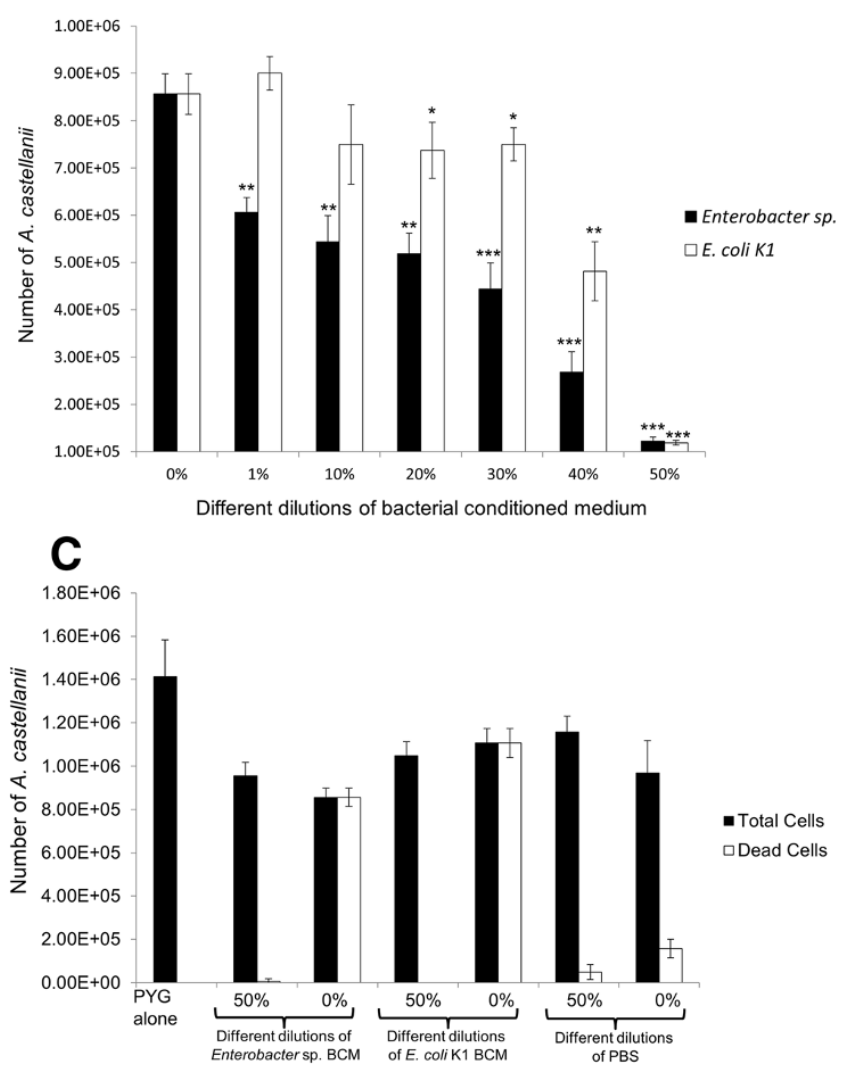

Figure 1 The conditioned media of Enterobacter sp. and Escherichia coli K1 exhibited amoebistatic and amoebicidal activities. (A) A. castellanii $\left(10^{5}\right)$ were incubated with $50 \%$ bacterial conditioned media (BCM) and $50 \% \mathrm{PYG}$ at $30^{\circ} \mathrm{C}$ for $48 \mathrm{~h}$. For controls, A. castellanii were incubated with PYG alone, 100\% PBS, and 50\% PBS plus 50\% PYG. After incubation, amoebae were counted using a haemocytometer. The initial inoculum of $10^{5}$ amoebae was used as baseline. Note that the incubation in the non-nutritive 100\% PBS showed no growth, while incubation in the growth medium resulted in over 8-fold increase in the number of amoebae compared with the original inoculum. P-values were calculated by comparing results of $100 \%$ PYG with BCM using student's t-test. (*) indicates $p<0.001$. (B) Enterobacter sp. and E. coli K1 BCM were diluted using PYG, where $0 \%$ dilution represents neat BCM, while $50 \%$ dilution represents BCM and PYG in 1:1 ratio. A. castellanii $\left(10^{5}\right)$ were incubated in various dilutions of BCM of Enterobacter sp. and E. coli K1 and incubated at $30^{\circ} \mathrm{C}$ for $48 \mathrm{~h}$. After incubation, amoebae were counted using a haemocytometer. The initial inoculum of $10^{5}$ amoebae increased to over 8-fold in PYG alone (0\% dilution). P-values were calculated by comparing results of 0\% BCM (i.e., PYG alone) with different concentrations of BCM. $\left({ }^{*}\right),\left(^{* *}\right)$ and $(* *)$ indicate $p$-values of $<0.05,<0.01$ and $<0.001$, respectively. (C) A. castellanii $\left(10^{6}\right)$ were incubated with neat $(0 \%)$ or diluted (50\%) BCM at $30^{\circ} \mathrm{C}$ for $24 \mathrm{~h}$. After incubation, $0.1 \%$ Trypan blue dye was added and amoebae along with the blue coloured amoebae (dead cells) were counted using a haemocytometer. Note that cells treated with neat BCM produced $A$. castellanii death. The data are presented as the mean \pm standard error of three independent experiments performed in duplicate. 
cut off Spin-X UF columns (Corning). Both the eluate and retentate were used in the aforementioned antimicrobial assays. To determine the chemical nature of active substance(s), conditioned media were treated with $1 \mathrm{mg}$ per $\mathrm{mL}$ pronase, mixture of broad spectrum proteases, at $37^{\circ} \mathrm{C}$ for $4 \mathrm{~h}$. Antimicrobial activity of pronase-treated conditioned media were determined as described above. Treatment of pronase did not block A. castellanii growth inhibitory activities of BCM (Figure 2A). To determine the approximate mass of active molecules, BCM were filtered through $10 \mathrm{kDa}$ and $5 \mathrm{kDa}$ size exclusion spin columns. The results revealed that eluate of both $5 \mathrm{kDa}$ and $10 \mathrm{kDa}$ exhibited $A$. castellanii growth suppression activities, suggesting that the active molecule(s) may be secondary metabolite(s) (Figure 2B).

Enterobacter sp. and E. coli K1 conditioned media are not cytotoxic to primary human brain microvascular endothelial cells

Cytotoxicity assays were performed as previously described [20]. Briefly, assays were performed in 24 well plates containing confluent human brain microvascular endothelial cells (HBMEC) monolayers. The conditioned media $(250 \mu \mathrm{L})$ or PYG medium $(250 \mu \mathrm{L})$ were added in $250 \mu \mathrm{L}$ RPMI-1640. Plates were incubated at $37^{\circ} \mathrm{C}$ in a $5 \% \mathrm{CO}_{2}$ incubator and monitored for monolayer disruptions over the period of $24 \mathrm{~h}$. After this incubation, the supernatants were collected from each well, centrifuged to remove cellular debris and then cytotoxic effects were determined by estimating the amount of lactate dehydrogenase release from HBMEC using a Cytotoxicity Detection kit (Roche Applied Sciences). The percent cytotoxicity was calculated as follows: \% cytotoxicity = (sample value - control value) / (total LDH release control value) $\times 100$. Control values were determined by incubating HBMEC monolayers with RPMI 1640 alone and total LDH release was obtained by completely lysing the HBMEC using 1\% Triton X-100. Cytotoxicity assays were performed to determine the effects of the Enterobacter sp., and E. coli $\mathrm{K} 1 \mathrm{BCM}$ on primary HBMEC. Neither E. coli K1 BMC nor Enterobacter sp. BCM produced host cell cytotoxicity $(0.3 \%$ and $2 \%$ host cell death respectively). As BCM was prepared using PYG, similar volume of PYG alone $(250 \mu \mathrm{L})$ were used as controls. The results revealed that PYG alone exhibited neither monolayer disruptions nor HBMEC cytotoxicity (ca. 2\%).

\section{A. castellanii conditioned media exhibited potent antibacterial activities against MRSA}

To determine the effects of ACM on multiple drug resistant (MDR) bacteria, clinical isolates of Acinetobacter sp., $P$. aeruginosa, MRSA and VRE were used. For antibacterial screening, approximately $10^{6}$ colony forming units (c.f.u), suspended in $10 \mu$ Lwere incubated with

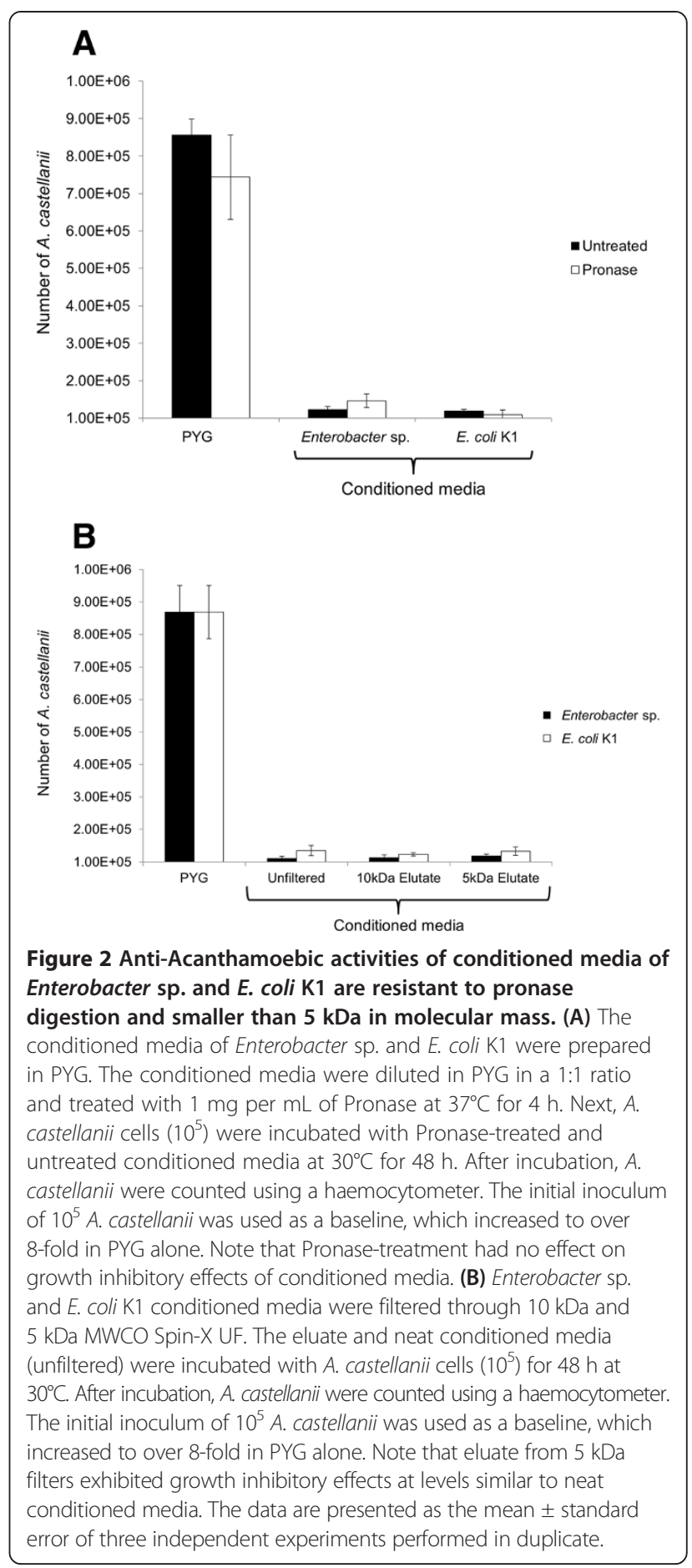

$190 \mu \mathrm{L}$ of $\mathrm{ACM}$ at $37^{\circ} \mathrm{C}$ for $18 \mathrm{~h}$. After incubation, cultures were ten-fold serially diluted in PBS and plated on agar plates. Plates were incubated at $37^{\circ} \mathrm{C}$, overnight and bacterial c.f.u. were enumerated [7]. For controls, bacteria were incubated in PYG alone and PBS alone. The results revealed that ACM killed more than 99\% MRSA and exhibited $8 \%$ bactericidal effects against VRE, but had no effect against other bacteria tested (Table 1). Heating at 
Table 1 Acanthamoeba conditioned medium (ACM) exhibited selective antibacterial properties

\begin{tabular}{|c|c|c|}
\hline Bacteria + Acanthamoeba conditioned medium (ACM) & $\begin{array}{l}\text { CFU remaining } \\
\text { (Bacteria + ACM) }\end{array}$ & Bacterial kill (\%) \\
\hline Methicillin-resistant Staphylococcus aureus (MRSA, $10 \mu \mathrm{L})+$ ACM (190 $\mu \mathrm{L})$ & 0 & 100 \\
\hline Acinetobacter $(10 \mu \mathrm{L})+\mathrm{ACM}(190 \mu \mathrm{L})$ & $1 \times 10^{10}$ & 0 \\
\hline Pseudomonas aeruginosa $(10 \mu \mathrm{L})+\mathrm{ACM}(190 \mu \mathrm{L})$ & $6 \times 10^{9}$ & 0 \\
\hline Vancomycin resistant Enterococcus faecalis (VRE, $10 \mu \mathrm{L})+\mathrm{ACM}(190 \mu \mathrm{L})$ & $3.75 \times 10^{5}$ & $8.12 \pm 9.8$ \\
\hline MRSA $(10 \mu \mathrm{L})+$ heated ACM $(190 \mu \mathrm{L})$ & 0 & 100 \\
\hline MRSA $(10 \mu \mathrm{L})+$ heated $\mathrm{ACM}(180 \mu \mathrm{L})+\mathrm{PYG}(10 \mu \mathrm{L})$ & $6.65 \times 10^{4}$ & $93.3 \pm 9.9$ \\
\hline MRSA $(10 \mu \mathrm{L})+$ heated ACM $(140 \mu \mathrm{L})+$ PYG $(50 \mu \mathrm{L})$ & $1.55 \times 10^{7}$ & 0 \\
\hline MRSA $(10 \mu \mathrm{L})+$ heated ACM eluate of $10 \mathrm{kDa}$ spin exclusion column $(190 \mu \mathrm{L})$ & 0 & 100 \\
\hline MRSA $(10 \mu \mathrm{L})+$ heated ACM eluatee of $5 \mathrm{kDa}$ spin exclusion column $(190 \mu \mathrm{L})$ & $1.34 \times 10^{10}$ & 0 \\
\hline 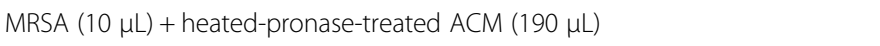 & 0 & 100 \\
\hline
\end{tabular}

The data are presented as the mean \pm SE of three independent experiments. ${ }^{a}$ An inoculum of $\sim 10^{6}$ bacterial c.f.u. was used.

$95^{\circ} \mathrm{C}$ for 10 min had no effect on the bactericidal properties of ACM. All subsequent experiments were performed using heated-ACM against MRSA. Serial dilutions showed that aliquots containing $190 \mu \mathrm{L}$ ACM exhibited $100 \%$ bactericidal effects, $180 \mu \mathrm{L}$ ACM showed $93.3 \%$ bactericidal effects, whereas dilution of 70\%: 30\% (ACM:PYG) had no bactericidal activity (Table 1). Antibacterial activity was observed in the eluate after passing ACM through $10 \mathrm{kDa}$ but retained by $5 \mathrm{kDA}$ size-exclusion spin columns, indicating that active ingredient(s) are between 5 to $10 \mathrm{kDa}$ in molecular mass. The treatment with pronase had no effect on bactericidal activity of ACM (Table 1). Notably, bacterial growth in PYG medium alone is comparable to LB (data not shown). The ACM had no cytotoxic effects on HBMECs in cytotoxicity assays (data not shown).

The presence of antimicrobials in microbes is not a novel concept and they are known to be an ancient weapon in the defense against the growth of other microbes. Since the discovery of penicillin produced in nature by mould, Penicillium in 1928 [8], a large number of microbes have been tested for the isolation of potentially useful antimicrobials, while there has been renewed interest in finding new antibiotics from unique sources such as actinomycetes, cyanobacteria and unculturable bacteria, in addition to known sources such as streptomycetes.

The ability of bacteria to thrive at high population densities and the predatory role of Acanthamoeba in the control of bacterial populations in the environment as well as the ability of the amoeba to act as a "Trojan horse" of the microbial world suggests that bacteriaprotists are involved in convoluted interactions [11-16]. The precise nature of such complex interactions is not clear, but it is shown here that both bacteria and amoebae are able to counter-attack each other and the overwhelming microbial densities by secreting/harboring antimicrobials. Among four bacteria tested, it is interesting that ACM exhibited selective and potent activity against MRSA. Superbugs, such as MRSA have shown the ability to cause untreatable infections and have become a major threat in our fight against bacterial infections [23]. The selective and potent nature of ACM against Gram-positive MRSA and partly against VRE, but not against Gramnegative $P$. aeruginosa is interesting. It is possible that $P$. aeruginosa and Acanthamoeba are involved in complex interactions where the bacteria can employ strategies to defend itself and counter amoebic attack [23]. Future studies will test ACM against a large panel of diverse bacterial pathogens to determine broad-spectrum or speciesspecific antibacterial properties. The discovery of antibacterial compound(s) from Acanthamoeba will open avenues for future studies in other free living amoebae/protists as a potential source of antibiotics for the treatment of emerging bacterial pathogens such as MRSA. Because of increasing trends in antibiotic resistance in different pathogenic bacteria [2], the present findings are promising in our efforts to treat infections caused by drug-resistant bacteria.

Similarly, among various bacterial isolates tested, BCM of Enterobacter sp. and E. coli $\mathrm{K} 1$ exhibited measurable amoebistatic and amoebicidal properties. Future studies will address the question of whether these $\mathrm{BCM}$ have selective action against Acanthamoeba or have wide scale activities against different protist pathogens. Interestingly, neither ACM nor BCM were cytotoxic to brain endothelial cells in vitro, suggestive of the selective action of these conditioned media against MRSA and Acanthamoeba, respectively. Future studies will further test the specificity and the absence of putative target(s) in human cells using primary cells of various origins. Overall, the present findings are promising in our search to find additional sources of antimicrobials with novel modes of action to confront this menace. Work is currently underway to characterize further the antimicrobial 
properties of $\mathrm{ACM}$ and $\mathrm{BCM}$ and active components, possibly novel secondary metabolites in the case of $\mathrm{BCM}$, being resistant to pronase and heat treatment and less than $5 \mathrm{kDa}$ in molecular mass. As antimicrobial resistance is on the increase, finding novel non-toxic antimicrobial compound(s) from a variety of species prevalent in clinical settings is crucial. It is hoped that these molecules will eventually be developed into treatments for bacterial and amoebic infections that are increasingly resistant to currently available drugs, however, this will require intensive research in the next few years.

\section{Competing interests}

The authors declare that they have no competing interests.

\section{Authors' contributions}

NK conceived the study. JI and RS designed and conducted all experiments under the supervision of NAK. RS, Jl and NAK contributed to the writing of the manuscript. All authors approved the final manuscript.

\section{Acknowledgements}

This work was supported by grants from the Aga Khan University.

Received: 30 October 2013 Accepted: 28 January 2014

Published: 30 January 2014

\section{References}

1. World Health Organization: Mortality Report. 2002. Available at http://www. who.org.

2. Alanis AJ: Resistance to antibiotics: are we in the post-antibiotic era? Arch Med Res 2005, 36:697-705.

3. Nordmann P, Naas T, Fortineau N, Poirel L: Superbugs in the coming new decade; multidrug resistance and prospects for treatment of Staphylococcus aureus, Enterococcus spp. and Pseudomonas aeruginosa in 2010. Curr Opin Microbiol 2007, 10:436-440.

4. Devasahayam G, Scheld WM, Hoffman PS: Newer antibacterial drugs for a new century. Expert Opin Investig Drugs 2010, 19:215-234.

5. Butler MS, Buss AD: Natural products-the future scaffolds for novel antibiotics? Biochem Pharmacol 2006, 71:919-929.

6. Demain AL: Antibiotics: natural products essential to human health. Med Res Rev 2009, 29:821-842.

7. Lee S, Duce I, Atkins H, Khan NA: Cockroaches and locusts: physicians' answer to infectious diseases. Int J Antimicrob Agents 2011, 37:279-280.

8. Fleming A: The story of penicillin. Am Inst Homeopath 1946, 39:154-157.

9. Tanaka YT, Mura SO: Agro active compounds of microbial origin. Annu Rev Microbiol 1993, 47:57-87.

10. De Jonckheere JF: Ecology of Acanthamoeba. Rev Infect Dis 1991 13(Suppl 5):S385-S387.

11. Khan NA: Acanthamoeba Biology and Pathogenesis. Norfolk, UK: Caister Academic Press; 2009.

12. Greub G, Raoult D: Microorganisms resistant to free-living amoebae. Clin Microbiol Rev 2004, 17:413-433.

13. Siddiqui $R$, Khan NA: War of the microbial worlds: who is the beneficiary in Acanthamoeba-bacterial interactions? Exp Parasitol 2012, 130:311-313.

14. Tamang MD, Kim S, Kim SM, Kong HH, Kim J: Interaction of Acinetobacter baumannii 19606 and 1656-2 with Acanthamoeba castellanii. J Microbiol 2011, 49:841-846.

15. Matz C, Moreno AM, Alhede M, Manefield M, Hauser AR, Givskov M, Kjelleberg S: Pseudomonas aeruginosa uses type III secretion system to kill biofilm-associated amoebae. ISME J 2008, 2:843-852.

16. Jousset A, Rochat L, Scheu S, Bonkowski M, Keel C: Predator-prey chemical warfare determines the expression of biocontrol genes by rhizosphereassociated Pseudomonas fluorescens. Appl Environ Microbiol 2010, 76:5263-5268.

17. Yousuf FA, Siddiqui R, Khan NA: Acanthamoeba castellanii is a potential environmental host for Enterobacter aerogenes and Aeromonas hydrophila. Parasit Vectors 2013, 6:169.
18. Siddiqui R, lqbal J, Maugeuret M, Khan NA: The role of Src kinase in the biological properties of Acanthamoeba. Parasit Vectors 2012, 5:112.

19. Sissons J, Kim KS, Stins M, Jayasekera S, Alsam S, Khan NA: Acanthamoeba castellanii induces host cell death via a phosphatidylinositol 3-kinasedependent mechanism. Infect Immun 2005, 73:2704-2708.

20. labal J, Rajani M, Siddiqui R, Khan NA: Neuropathogenic Escherichia coli K1 does not exhibit proteolytic activities to exert its pathogenicity. J Negat Results Biomed 2013, 12:8.

21. Cardas M, Khan NA, Alsam S: Staphylococcus aureus exhibit similarities in their interactions with Acanthamoeba and ThP1 macrophage-like cells. Exp Parasitol 2012, 132:513-518.

22. Baig AM, Iqbal J, Khan NA: In vitro efficacies of clinically available drugs against growth and viability of an Acanthamoeba castellanii keratitis isolate belonging to the T4 genotype. Antimicrob Agents Chemother 2013, 57:3561-3567.

23. Pantosti A, Venditti M: What is MRSA? Eur Respir J 2009, 34:1190-1196.

doi:10.1186/1756-3305-7-56

Cite this article as: lqbal et al: Acanthamoeba and bacteria produce antimicrobials to target their counterpart. Parasites \& Vectors 2014 7:56.

\section{Submit your next manuscript to BioMed Central and take full advantage of:}

- Convenient online submission

- Thorough peer review

- No space constraints or color figure charges

- Immediate publication on acceptance

- Inclusion in PubMed, CAS, Scopus and Google Scholar

- Research which is freely available for redistribution 